\title{
Never, always and maybe. Addressing attitudes of patients towards emergency medicine research
}

\author{
Michelle Biros
}

As the clinical specialties of emergency medicine and prehospital medicine developed, patients who otherwise would have died in the field began to survive to reach the ED. It soon became apparent that existing emergent treatments for many critical conditions were unsatisfactory and/or were understudied. However, traditional methods of obtaining prospective informed consent for research participation are not feasible when a devastating pathology deprives patients of meaningful decision- making capacity. With that in mind, a set of regulations was developed by the Federal regulatory agencies in USA; this allows for exception from informed consent (EFIC) in emergency research in specific clinical circumstances ${ }^{1}$ and went into effect in 1996. The emergency medicine community was the key driver in the creation of these regulations. $^{23}$

The criteria for EFIC studies are well defined and are briefly summarised in box 1 . Among these requirements is prestudy community consultation, aimed at

\begin{abstract}
'providing the opportunity for discussions with and soliciting opinions from the community(ies) in which the study will take place (geographic area) and from which the study subjects will be drawn (similar demographics of patients with the emergent condition under study). These communities may not be the same; when they are not the same, both communities should be consulted...'.1
\end{abstract}

It is an opportunity to allow information exchange, ensures that the community's concerns are known when the institutional review boards (IRBs) and ethics committees engage in decision-making about potential EFIC studies and allows investigators to consider information related to the study that may not otherwise have been apparent to the scientific community.

Many types of community consultations have been employed, such as presentations at existing public forums,

Correspondence to Dr Michelle Biros, Department of Emergency Medicine, University of Minnesota Medical School, Minneapolis, MN 55415, USA;

biros001@umn.edu individual interviews and surveys, and specific groups convened for the specific purpose of community consultation. Best practices for community consultation are not known, but all methods require a two-way communication between the investigator and the participant. Box 2 includes the information required to be discussed during community consultation and the responsibilities of IRBs based on the results of community consultation.

By definition, patients who qualify for EFIC studies lack autonomy; a long and embarrassing history of proven scientific misconduct and abuse of vulnerable patients make us all sensitive to the incredible responsibility this poses on investigators. It is unclear if community consultation does in fact add human subject protection, but it has provided insight into patient populations, and the opportunity to determine how emergency research, with and without consent, is viewed by the population. ${ }^{45}$

The study by Buckley et $a l^{6}$ queried specific human factor domains to assess the attitudes towards and motivations for emergency research participation in a group of patients admitted with emergent medical and surgical conditions. Trust in the medical enterprise, concerns about personal well-being and altruism are important aspects in the acceptance of enrolment into emergency research trials, while concerns over the violation of autonomy made some more likely to be apprehensive about such research. These observations make sense and in fact collaborate other research around such concerns. $^{4}$ 7-9

Of particular interest and importance to EFIC research is other observationsthat complex concepts are difficult to understand, some patients may have trouble distinguishing research from clinical practice and some patients are eager to please and seek affirmation when directly communicating with an investigator.

These findings confirm an observation I personally have made after applying the EFIC regulations in my own research, when trying to derive metrics to determine their effectiveness, when consulting with other EFIC researchers and in observing many community consultation activities. When attitudes towards emergency research in general and EFIC in particular are assessed, I have observed that three types of respondent groups often emerge.

The first is a group of individuals who would always agree to research. Their motivation is based on a number of factors, such as the desire to do good and contribute, inability to distinguish research from clinical care, trust that the investigator wants only what is best for them and/ or insecurity about their own ability to make a determination, therefore leaving it in the hands of a trusted representative (ie, the investigator). They may be concerned about missing an opportunity if they do not agree or that refusal of research in some way impacts their clinical care. The second group is comprised of those who would never agree to research under any circumstance. This could be based on previous negative experiences, concerns about lack of control and lack of autonomy, or general distrust of the scientific enterprise. The final group is uncertain or undecided about the worthiness of research participation. These individuals may have had a variable experience in the past with medicine, may be reluctant to make their true feelings known and/or may not completely understand the complex concepts involved in discussion of emergency research.

Each group poses specific challenges to and imposes unique responsibilities on

Box 1 Major criteria for exception from informed consent (EFIC) requirements summarised

- Condition under study is devastating and currently available treatments are unproven or unsatisfactory

- Time is critical for application of the study intervention and the research cannot be done without an EFIC

- Promising preclinical trials that suggest potential benefits of the investigation exist

- It is not feasible to prospectively identify and consent potential subjects

- There is a reasonable risk-benefit ratio

- The research has the potential to directly benefit the study subject

- Community consultation has occurred before the study begins Public disclosure of study information occurs before, during and after the study 
Box 2 Investigator and

institutional review board (IRB)

responsibilities in community

consultation

At a minimum, the investigator must:

- Discuss the exception from informed consent (EFIC) plan (ie, methods for community consultation, etc.) with the IRB/research committee prior to its implementation

- Establish an informed consent process in the event that prospective consent (ie, surrogate) can be obtained for some subjects

- Indicate to the community that informed consent will not be obtained in most subjects

- Disclose the risks and benefits of research participation

- Indicate that there is an individual right to refuse participation

- Indicate how subjects wishing to be excluded can indicate this preference IRBs must:

- Ensure the EFIC plan provides an unbiased method for feedback and approve the EFIC plan

- Decide if community consultation has been adequately performed and includes the correct communities

- Consider all community discussions and concerns

- Document IRB discussion and resolutions of community raised controversies

- Decide if the study can go forward, requires revision for implementation within the local community or cannot be conducted within the local community

critical care researchers. A different approach is required to address the needs of each group when fulfilling the requirements of patient enrolment into EFIC trials. The requirement of community consultation for EFIC has offered insight into these groups and potential methods to fulfil these unique responsibilities.

It would be easy and convenient for researchers to minimise the risks involved and downplay the fact that autonomy is not possible in EFIC research to those individuals who would always agree to research participation. The integrity of the researcher must remain very high to ensure the risks and benefits are accurately described, and that these individuals truly understand the study and their right to question without consequence.

Researchers often attempt to 'convert' those individuals who would never agree to research. With such individuals, the EFIC investigator's responsibilities include determining the motivation for refusal and providing, in an unbiased and unemotional way, the information to ensure that the refusal of research participation is truly informed. If legitimate concerns are voiced after these interventions, the researcher must consider these objections and bring them to the attention of the ethics committee that is charged with research oversight. While community consultation is not community consent or community veto of the study, the final decision to begin an EFIC study is informed by valid community concerns. If, on the other hand, after adequate communication regarding research has been made, the concerns voiced by members of this group are not substantial, and the refusal represents a personal belief system, little can be done to change the mind of these persons. Perhaps the energy spent trying to convince them should be directed to communication with the third group-those who are uncertain or undecided.

The investigator's responsibilities with the uncertain group are the most intensive. The concerns that cause uncertainty need to be determined. When non-commitment is due to lack of understanding, researchers must be sure that complex research concepts are carefully explained; describing the concepts in the context of a specific study has been seen to improve understanding. ${ }^{4} 5$ If the uncertainty is due to concerns about losing the connection to their clinician and care plans, the investigator must delineate the difference between clinical care and research. If the uncertainty is due to reluctance to voice personal beliefs in a public forum, the investigator can establish a one-on-one dialogue with the individual. Although more labour intensive, this is a productive way to develop the connection needed to allow an honest discussion of core beliefs. ${ }^{10} 11$

Understanding community attitudes allows emergency investigators to strategise best methods to address community concerns regarding emergency research without consent. The article by Buckley et $a l^{6}$ adds to our current knowledge. Combined with previous studies, this informs investigators on their approach to engaging the community in emergency research.

Competing interests None declared.

Provenance and peer review Not commissioned; internally peer reviewed.

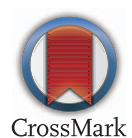

To cite Biros M. Emerg Med J 2016;33:374-375.

Accepted 19 February 2016

Published Online First 11 March 2016

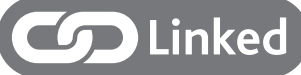

http://dx.doi.org/10.1136/emermed-2015-205146

Emerg Med J 2016;33:374-375.

doi:10.1136/emermed-2016-205811

\section{REFERENCES}

1 US Food and Drug Administration 21 CFR 50.24. Protection of human subjects.

2 Biros MH, Runge JW, Lewis RJ, et al. Emergency medicine and the development of the food and drug administration's final rule on informed consent and waiver of informed consent in emergency research circumstances. Acad Emerg Med 1998;5:359-68.

3 Biros MH, Lewis RJ, Olson CM, et al. Informed consent in emergency research: consensus statement from the coalition conference of acute resuscitation and critical care researchers. JAMA 1995:273:1283-7.

4 Biros MH, Sargent C, Miller K. Community attitudes towards emergency research and exception from informed consent. Resuscitation 2009;80:1382-7.

5 Biros MH. Does community consultation matter? Acad Emerg Med 2013;20:104-5.

6 Buckley JM, Irving AD, Goodacre S. How do patients feel about taking part in clinical trials in emergency care? Emerg Med J 2016;33:376-80.

7 Dickert NW, Scicluna VM, Baren JM, et al. Patients perspectives of enrollment in research without consent: the patients' experiences in emergency research-progesterone for the treatment of traumatic brain injury study. Crit Care Med 2015;43:603-12.

8 Dickert NW, Mah VA, Baren JM, et al. Enrollment in research under exception from informed consent: the Patients' Experiences in Emergency Research (PEER) Study. Resuscitation 2013;84:1416-21.

9 Dickert NW, Kass NE. Patients' perceptions of research in the emergency settings; a study of survivors of sudden cardiac death. Soc Sci Med 2009;68:183-91.

10 Mah V, Dickert NW, Biros MH, et al. The method matters: community consultation methods influence attitudes and understanding of proposed trial using exception from informed consent for research in emergency settings. Circulation 2012;126(21 Suppl):A149.

11 Dickert NW, Mah VA, Biros MH, et al. Consulting communities when patients cannot consent: a multicenter study of community consultation for research in emergency settings. Crit Care Med 2014;42:272-80. 